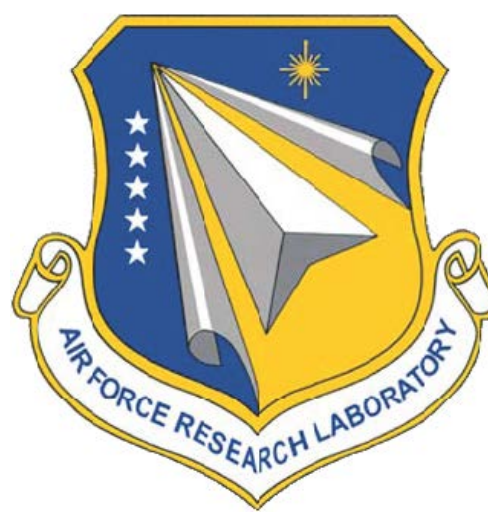

\title{
Development of Oculometrics for Operational Based Vision Assessment
}

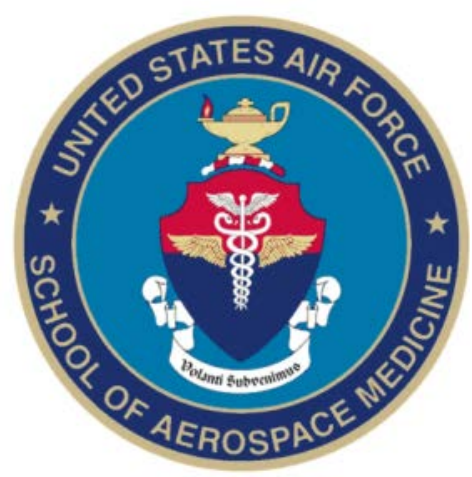

Leland S. Stone, Dorion B. Liston

Distribution A: Approved for public release; distribution is unlimited. Case Number: 88ABW-2014-1794, 18 Apr 2014
Air Force Research Laboratory $711^{\text {th }}$ Human Performance Wing School of Aerospace Medicine Aerospace Medicine Department Ophthalmology Branch 2510 Fifth St. Wright-Patterson AFB, OH 45433-7913 


\section{NOTICE AND SIGNATURE PAGE}

Using Government drawings, specifications, or other data included in this document for any purpose other than Government procurement does not in any way obligate the U.S. Government. The fact that the Government formulated or supplied the drawings, specifications, or other data does not license the holder or any other person or corporation or convey any rights or permission to manufacture, use, or sell any patented invention that may relate to them.

Qualified requestors may obtain copies of this report from the Defense Technical Information Center (DTIC) (http://www.dtic.mil). PUBLICATION IN ACCORDANCE WITH ASSIGNED DISTRIBUTION STATEMENT.

//SIGNATURE//

LEROY G. BEYER, Col, USAF, MC, CFS Chief, Clinical Sciences Division
//SIGNATURE//

CHUNG SIEDLECKI, Col, USAF, MC, CFS Chair, Aerospace Medicine

This report is published in the interest of scientific and technical information exchange, and its publication does not constitute the Government's approval or disapproval of its ideas or findings. 


\section{REPORT DOCUMENTATION PAGE}

Public reporting burden for this collection of information is estimated to average 1 hour per response, including the time for reviewing instructions, searching existing data sources, gathering and

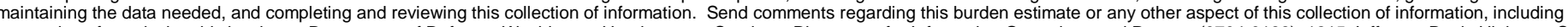

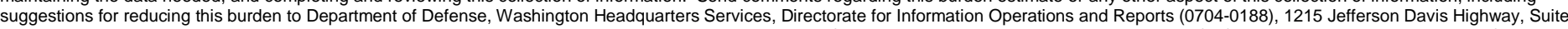
1204, Arlington, VA 22202-4302. Respondents should be aware that notwithstanding any other provision of law, no person shall be subject to any penalty for failing to comply with a collection of information if it does not display a currently valid OMB control number. PLEASE DO NOT RETURN YOUR FORM TO THE ABOVE ADDRESS.
1. REPORT DATE (DD-MM-YYYY)
2. REPORT TYPE
3. DATES COVERED (From - To)
22 Jan 2014
Special Report
June 2012 - June 2013

\section{TITLE AND SUBTITLE}

5a. CONTRACT NUMBER

Development of Oculometrics for Operational Based Vision Assessment

5b. GRANT NUMBER

5c. PROGRAM ELEMENT NUMBER

6. AUTHOR(S)

Leland S. Stone, Dorion B. Liston 5d. PROJECT NUMBER

5e. TASK NUMBER

5f. WORK UNIT NUMBER

8. PERFORMING ORGANIZATION REPORT NUMBER

AFRL-SA-WP-SR-2014-0005

10. SPONSORING/MONITOR'S ACRONYM(S)

11. SPONSOR/MONITOR'S REPORT NUMBER(S)

\section{DISTRIBUTION / AVAILABILITY STATEMENT}

Distribution A: Approved for public release; distribution is unlimited. Case Number: 88ABW-2014-1794, 18 Apr 2014

\section{SUPPLEMENTARY NOTES}

\section{ABSTRACT}

This project is part of a larger program conducted at the USAF School of Aerospace Medicine's (USAFSAM) Operational Based Vision Assessment (OBVA) laboratory to determine whether an individual's motion sensitivity is predictive of their operational performance. The Air Force does not currently have a standard method to measure an individual's motion sensitivity and a first step was to research the feasibility of using such an approach. Previous research with the University of Birmingham used perceptual tests to estimate the distribution of motion thresholds, found a significant range of thresholds in the normally-sighted population and demonstrated that scores on these tests are predictive of driving performance.

In the effort described here, USAFSAM defined project objectives and funded NASA's Ames Research Center (ARC) to investigate an alternative method that uses eye movement measurements (oculometrics), which were developed at ARC, to estimate an individual's motion sensing capability. This final report describes their work. NASA-ARC conducted experiments in their laboratory and delivered oculometric data for a normally-sighted cohort of 40 subjects.

\section{SUBJECT TERMS}

Visual motion processing, motion perception, eye movements, eye tracking, oculometrics, vision standards

16. SECURITY CLASSIFICATION OF:

a. REPORT

$\mathrm{U}$ b. ABSTRACT

$\mathrm{U}$

\begin{tabular}{c|c}
$\begin{array}{c}\text { 17. LIMITATION } \\
\text { OF ABSTRACT }\end{array}$ & $\begin{array}{c}\text { 18. NUMBER } \\
\text { OF PAGES }\end{array}$ \\
SAR & 18
\end{tabular}

19a. NAME OF RESPONSIBLE PERSON Marc Winterbottom 19b. TELEPHONE NUMBER (include area code) 
This page intentionally left blank. 


\section{TABLE OF CONTENTS}

Section

Page

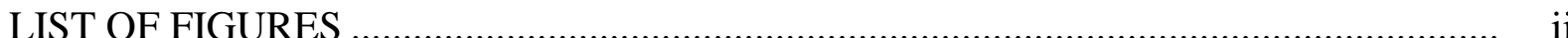

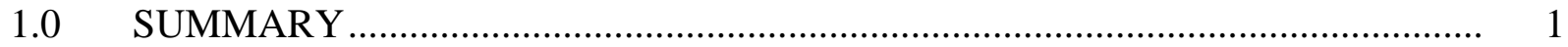

$2.0 \quad$ BACKGROUND AND OVERVIEW ................................................................ 1

2.1 Hypotheses .................................................................................................

2.2 Specific Aims ................................................................................................... 1

3.0 METHODS AND SYSTEM ENGINEERING SPECIFICATIONS ........................... 2

3.1 Rashbass Arrows-In Task ................................................................................ 2

3.2 Eye-Movement Recording ............................................................................. 2

3.3 Pursuit Analyses............................................................................................ 2

4.0 RESULTS AND BASELINE PERFORMANCE DATA ………………………...... 3

4.1 Defining the OMCAT Oculometric Measures....................................................... 3

4.2 Summary of Individual Data and Across-Trial Variability .................................. 4

4.3 Baseline Population Oculometrics and Across-Subject Variability .................... 5

4.3.1 Initiation Metrics................................................................................. 5

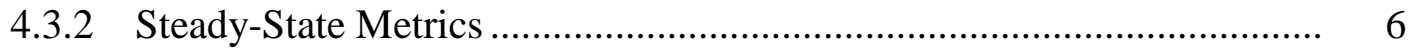

4.3.3 Direction-Tuning Metrics ................................................................... 7

4.3.4 Speed-Tuning Metrics.............................................................................. 8

4.4 Reliability and Across-Time Variability.............................................................. 8

4.5 Correlation .............................................................................................. 10

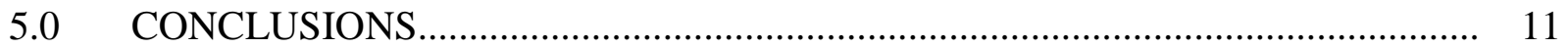

6.0 REFERENCES .............................................................................. 11

LIST OF ABBREVIATIONS AND ACRONYMS _.................................................. 12 


\section{LIST OF FIGURES}

Figure

Page

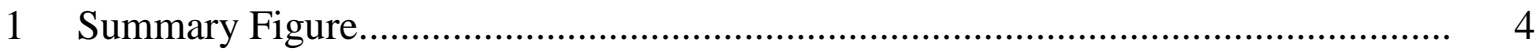

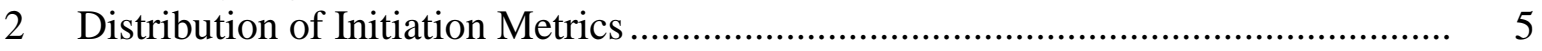

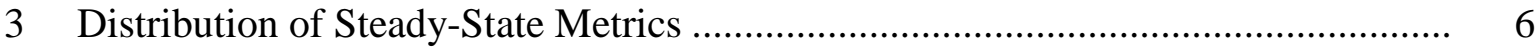

4 Distribution of Direction-Tuning Metrics............................................................ 7

5 Distribution of Speed-Tuning Metrics .......................................................... 8

6 Test-Retest Statistics for 8 Oculometric Measures for All 6 Subjects Tested in this Control Experiment ...................................................................... 9

7 Correlation Statistics for All Pairs of Our 10 Oculometric Measures Across All 40 Subjects Tested ............................................................................ 10 


\subsection{SUMMARY}

In this project, we designed and validated a new concept for an Oculometric Motion Processing Capability Assessment Tool (OMCAT) suitable for quickly and reliably assessing the visual motion processing capabilities of airmen. We also demonstrated that the human performance characteristics captured by OMCAT are not captured by acuity measures. The delivered OMCAT framework is ready for development and integration into a prototype system to enable the assessment of the OMCAT suite of oculometric measures as quantitative predictors of pilot performance in simulator tests in support of Air Force vision screening applications.

\subsection{BACKGROUND AND OVERVIEW}

\subsection{Hypotheses}

- Hypothesis \#1:

a. A small set of oculometric measures can be found to succinctly characterize the visual motion processing capabilities of individual pilots, of their cohort, and of an aged-matched normal population, and

b. These oculometric measures can be used to detect visual motion processing performance either above or below normal.

- Hypothesis \#2: Some of our oculometric measures will correlate with operational performance in OBVA tasks. Note that this hypothesis will be tested in the OBVA laboratory and is not part of the deliverable for this project.

\subsection{Specific Aims}

- Tailor NASA Ames's current laboratory tasks into a single task to yield a 10-minute "clinical" test

- Develop analyses of smooth tracking (pursuit) eye movements to measure speed and direction discrimination

- Validate a small set of performance measures to provide repeatable and independent performance metrics

- Provide a database of baseline human performance

- Establish engineering specifications for a future OMCAT system from our hardware prototyping efforts 


\subsection{METHODS AND SYSTEM ENGINEERING SPECIFICATIONS}

\subsection{Rashbass Arrows-In Task}

We used a two-dimensional variant of the Rashbass step-ramp paradigm [1]. Subjects began each trial by fixating the tracking target (a small red spot) in primary position and pressing a mouse button. Subjects then fixated the red spot for a randomized duration drawn from an exponential distribution [2] (mean: $700 \mathrm{~ms}$, minimum: $200 \mathrm{~ms}$, maximum: $5000 \mathrm{~ms}$ ) to defeat possible temporal expectation of motion onset. After the randomized duration had elapsed, the tracking spot made a small step [3] in a random direction drawn from a uniform distribution around the unit circle (in 2-degree increments) and immediately began moving back toward the initial fixation location. The step size was set such that the target crossed its original fixation location $200 \mathrm{~ms}$ after motion onset. On each trial, the velocity of the target spot was drawn from a distribution of five speeds (16, 18, 20, 22, $24 \mathrm{deg} / \mathrm{s})$ and lasted 1.2 seconds. Each experiment consisted of 180 tracking trials ( 15 minutes) to maintain the subject's alertness [4] and allow for a clinically reasonable behavioral session. Stimuli were displayed on a 60-Hz CRT with resolution of 1024 by 768 pixels at a viewing distance of $57 \mathrm{~cm}$, which subtended an area of 38 degrees horizontal by 29 degrees vertical.

\subsection{Eye-Movement Recording}

We sampled eye position at $240 \mathrm{~Hz}$ with an ISCAN eye-tracker (ISCAN Inc., Burlington, MA). The eye-tracker camera uses a 256- by 256-pixel image and returns the centroid of the pupil rounded to the nearest pixel. The eye-position traces were calibrated with six parameters (gain, offset, and cross-terms for horizontal and vertical [5]) fit to the raw digital values for fixations at nine screen locations, which yielded a precision of better than $0.3^{\circ}$ (standard deviation of eye-position while fixating). Saccades were detected by taking the correlation between a saccade-shaped velocity template and the horizontal eye velocity trace [6], which we then excised from the velocity traces during subsequent analyses.

\subsection{Pursuit Analyses}

We used a "hinge" model [7] to mark the onset of the pursuit movement. Because our tracking target was a single stimulus moving in a random direction, we marked pursuit onset using velocity parallel to target motion. The hinge consists of two line segments (baseline and response, each $100 \mathrm{~ms}$ in duration) occurring consecutively; pursuit latency was defined as the point at which the two line segments intersected. We added three constraints to the algorithm to increase its robustness. We defined the baseline velocity to be zero, constrained the acceleration to be positive, and modified the error function by a "recinormal" prior probability distribution. For each trial, our algorithm measured the error between the best fit of the two free parameters describing the hinge model (latency and acceleration) to the velocity trace over the interval from 100 to $400 \mathrm{~ms}$. This error function was then weighted by a recinormal [8] function (mean: $5.4 \mathrm{~s}^{-}$ 1 , standard deviation $2 \mathrm{~s}^{-1}$ ) with its median at the expected pursuit latency of $185 \mathrm{~ms}$. Pursuit latency was defined to be the time that yielded the minimum error; open-loop acceleration was defined to be the slope of the response interval. Steady-state gain was defined to be the ratio of radial eye velocity to target velocity over the interval from 400 to $700 \mathrm{~ms}$ after motion onset, and 
the proportion of smooth pursuit was defined to be the ratio of eye displacement during smooth pursuit to total eye displacement over this interval. We also measured the radial direction of the pursuit response during the steady-state interval to quantify the signal and noise properties of the pursuit response to angular motion as described in detail previously [1].

\subsection{RESULTS AND BASELINE PERFORMANCE DATA}

\subsection{Defining the OMCAT Oculometric Measures}

We established four different categories of oculometric performance parameters.

1. Initiation (INIT) measures: After detecting and removing saccadic intrusions from the eye-velocity traces [6], we measured the initial latency (INIT latency) of the pursuit response (in $\mathrm{ms}$ ) with a best-fitting hinge function [7]. We also quantified the initial mean acceleration (INIT acc) in the first $100 \mathrm{~ms}$ of the smooth eye-movement response (the so-called open-loop interval) [9]. These provide measures of the vigor of the initial pursuit visual-tracking response.

2. Steady-state (SS) measures: All measures of steady-state tracking were made in the interval from 400 to $700 \mathrm{~ms}$ following motion onset. We measured the ratio of the steady-state eye-speed to target-speed, or the traditional steady-state gain (SS gain) [3], the mean size of the catch-up saccades (SS sacc amp), and the proportion of smooth tracking (SS prop smooth).

3. Direction-tuning (DIR) measures: We also measured the directional tuning of the pursuit response [1]. This provided a measure of the oblique effect (DIR anisotropy), horizontalvertical asymmetry (DIR asymmetry), and directional uncertainty (DIR noise).

4. Speed-tuning (SPD) measures: We also developed a new method for simultaneously measuring speed tuning during the same task. This provided two new oculometric measures: speed responsiveness (SPD slope) and speed uncertainty (SPD noise).

The pursuit initiation and steady-state tracking measures characterize the trial-by-trial tracking response dynamics, whereas the direction and speed-tuning measures characterize the signal and noise components of the response to changes in target direction and speed. Two of the three direction-tuning measures are unique in that they capture nonlinear distortions as opposed to properties of a quasi-linear response. 


\subsection{Summary of Individual Data and Across-Trial Variability}

Figure 1 shows all the oculometric data for a single observer from a single test session. The distributions show that the measures are well behaved (pseudo-Gaussian). The direction tuning shows a well-behaved sensitivity to target direction along with the typical oblique effect documented previously [1]. The speed tuning documents a well-behaved sensitivity to target speed. Figure 1 is formatted as a summary output data figure for a 15-minute clinical test.
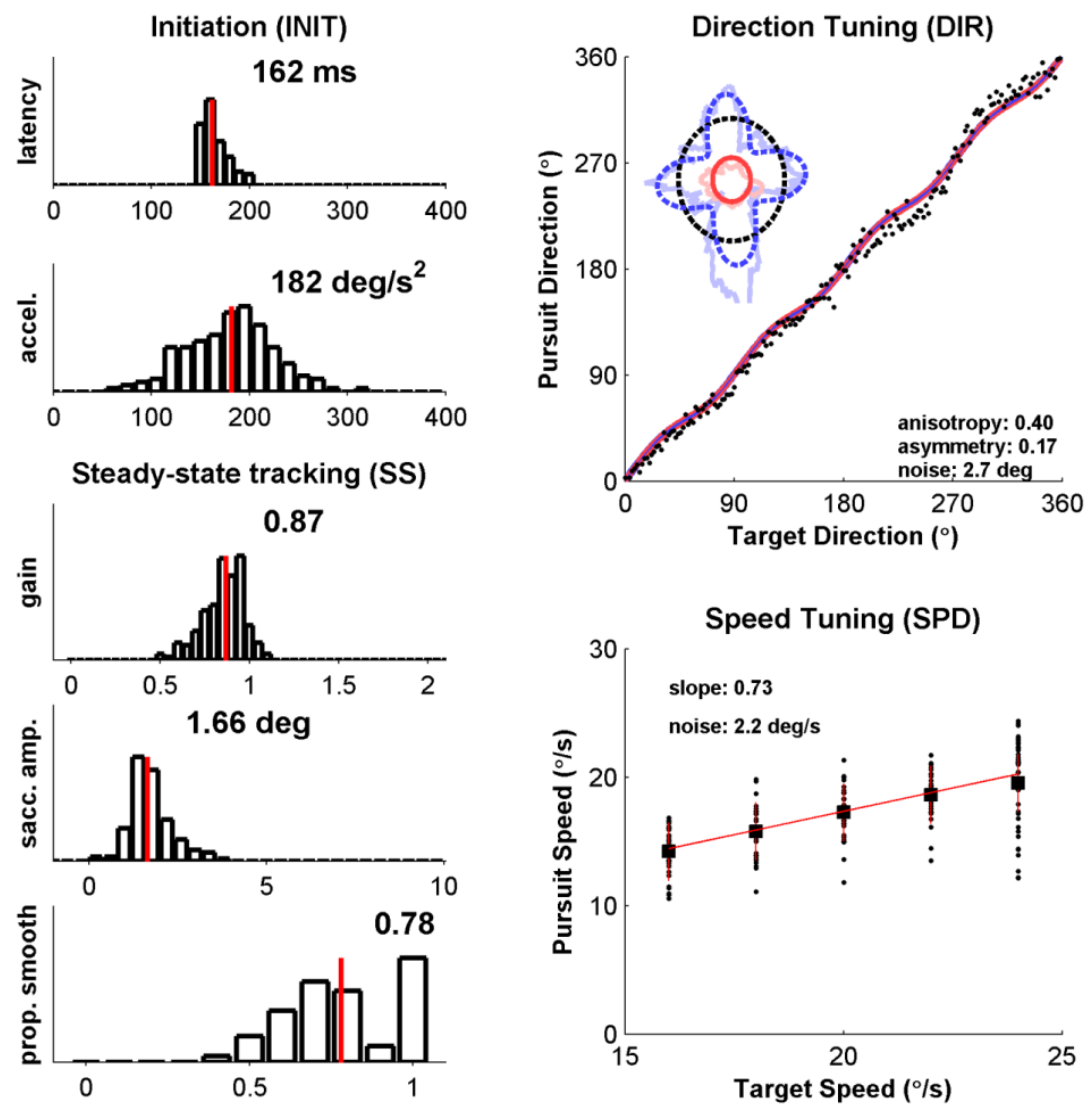


\subsection{Baseline Population Oculometrics and Across-Subject Variability}

\subsubsection{Initiation Metrics (Figure 2)}

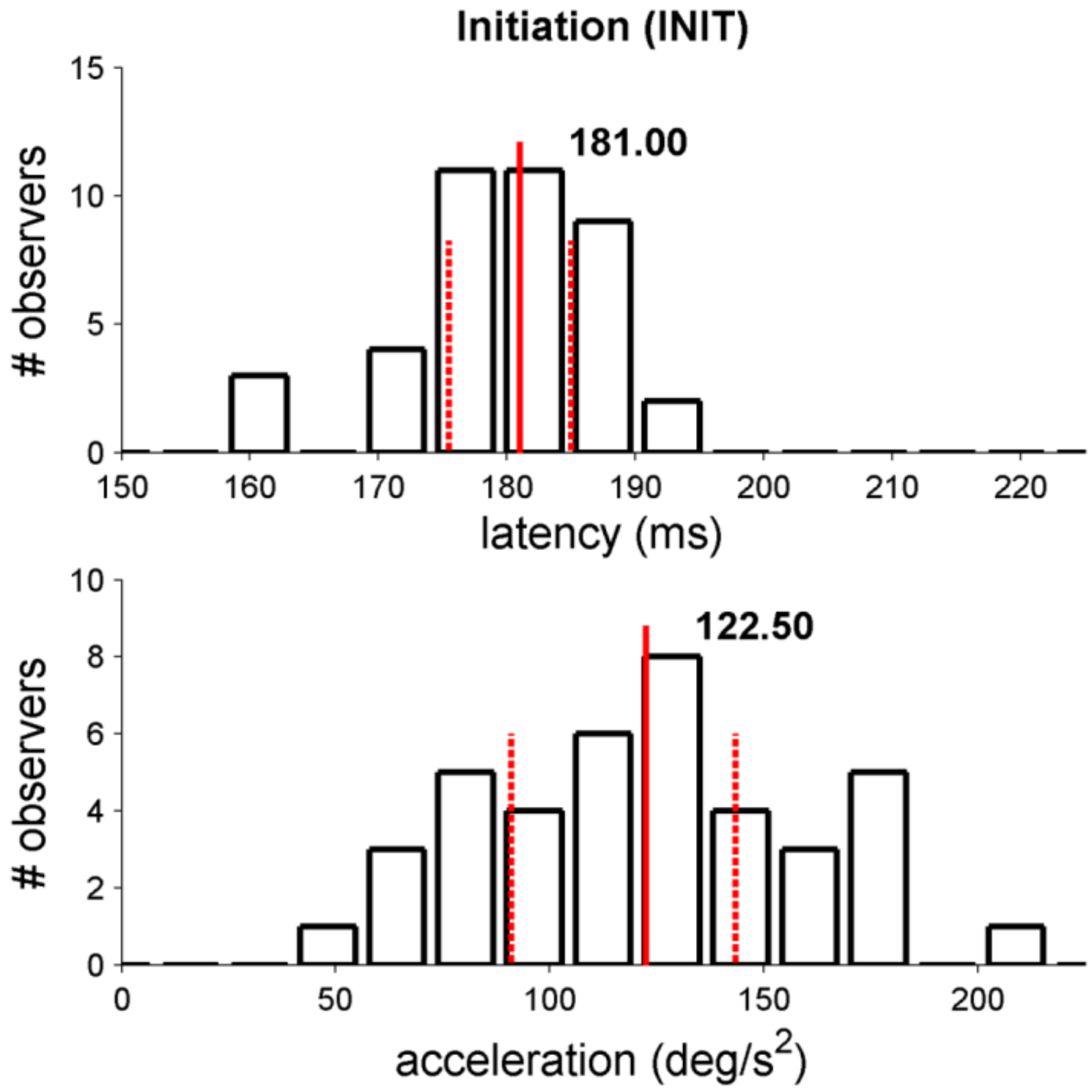

Figure 2. Distribution of Initiation Metrics. The median latency of $181 \mathrm{~ms}$ and the median acceleration of $122.5 \mathrm{deg} / \mathrm{s}^{2}$ are indicated by the solid vertical red line. Dashed lines indicate the quartiles. Note the well-behaved (pseudo-Gaussian) nature of the distributions. 


\subsubsection{Steady-State Metrics (Figure 3)}

\section{Steady-State Tracking (SS)}
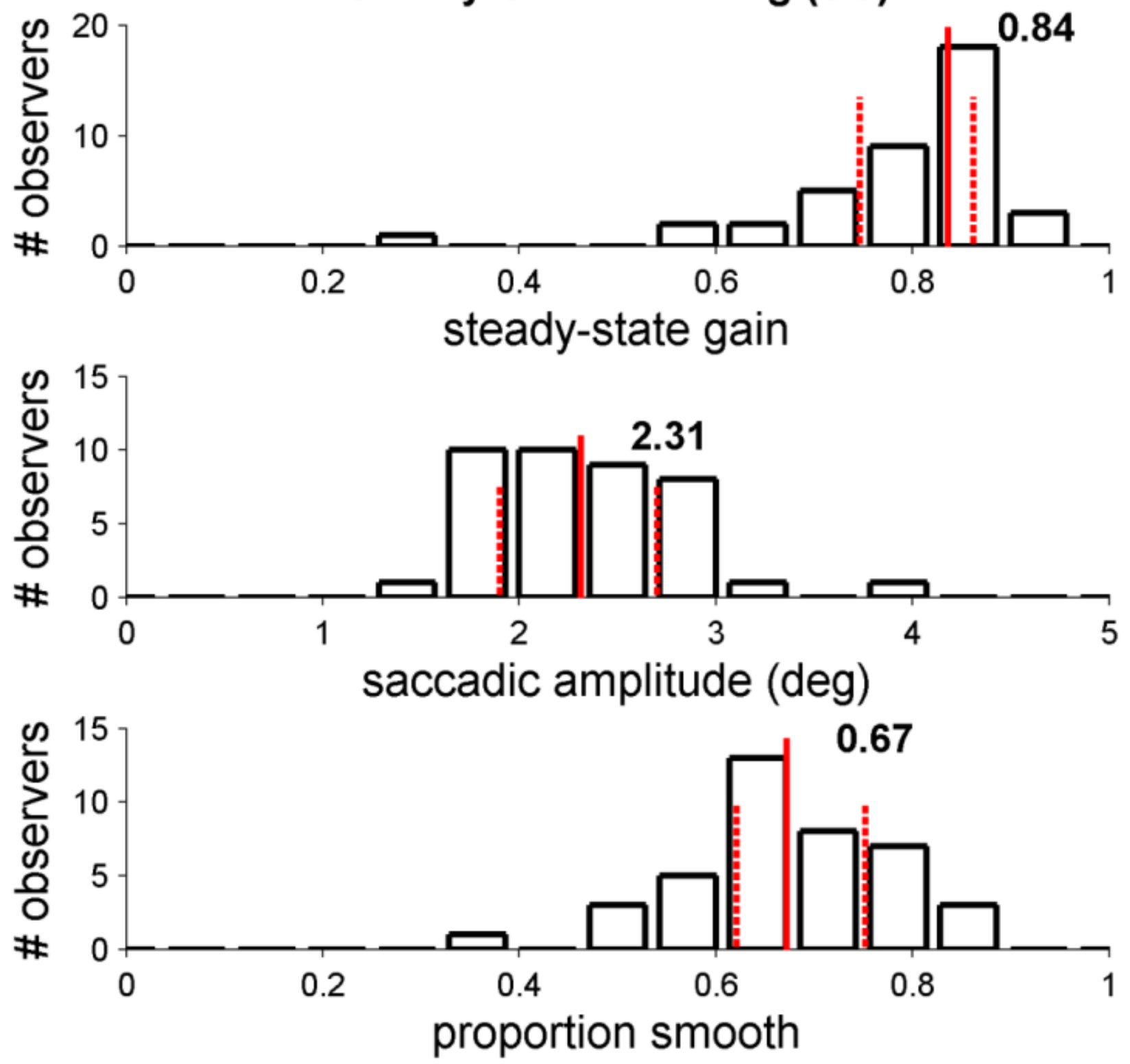


\subsubsection{Direction-Tuning Metrics (Figure 4)}
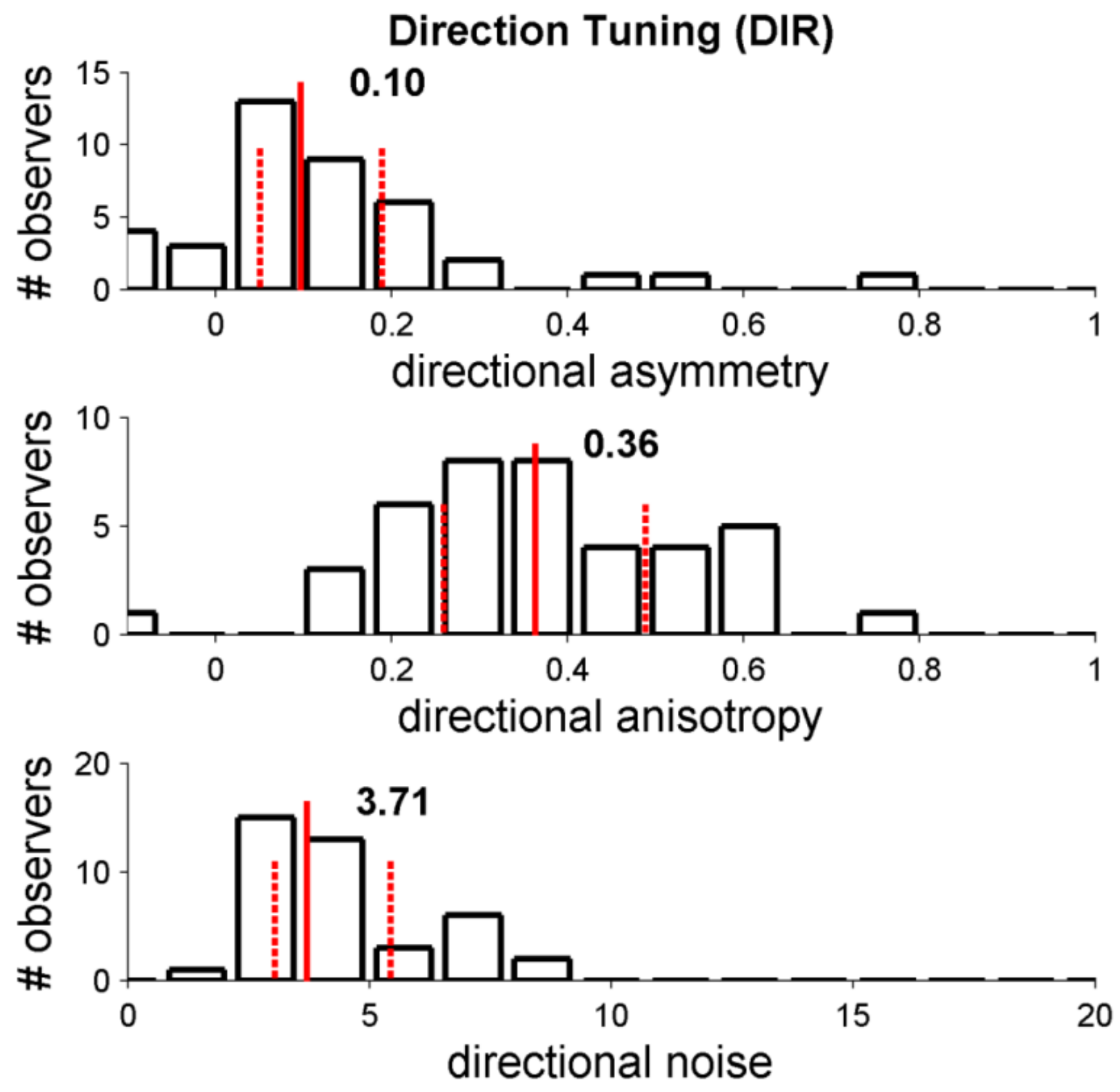


\subsubsection{Speed-Tuning Metrics (Figure 5)}
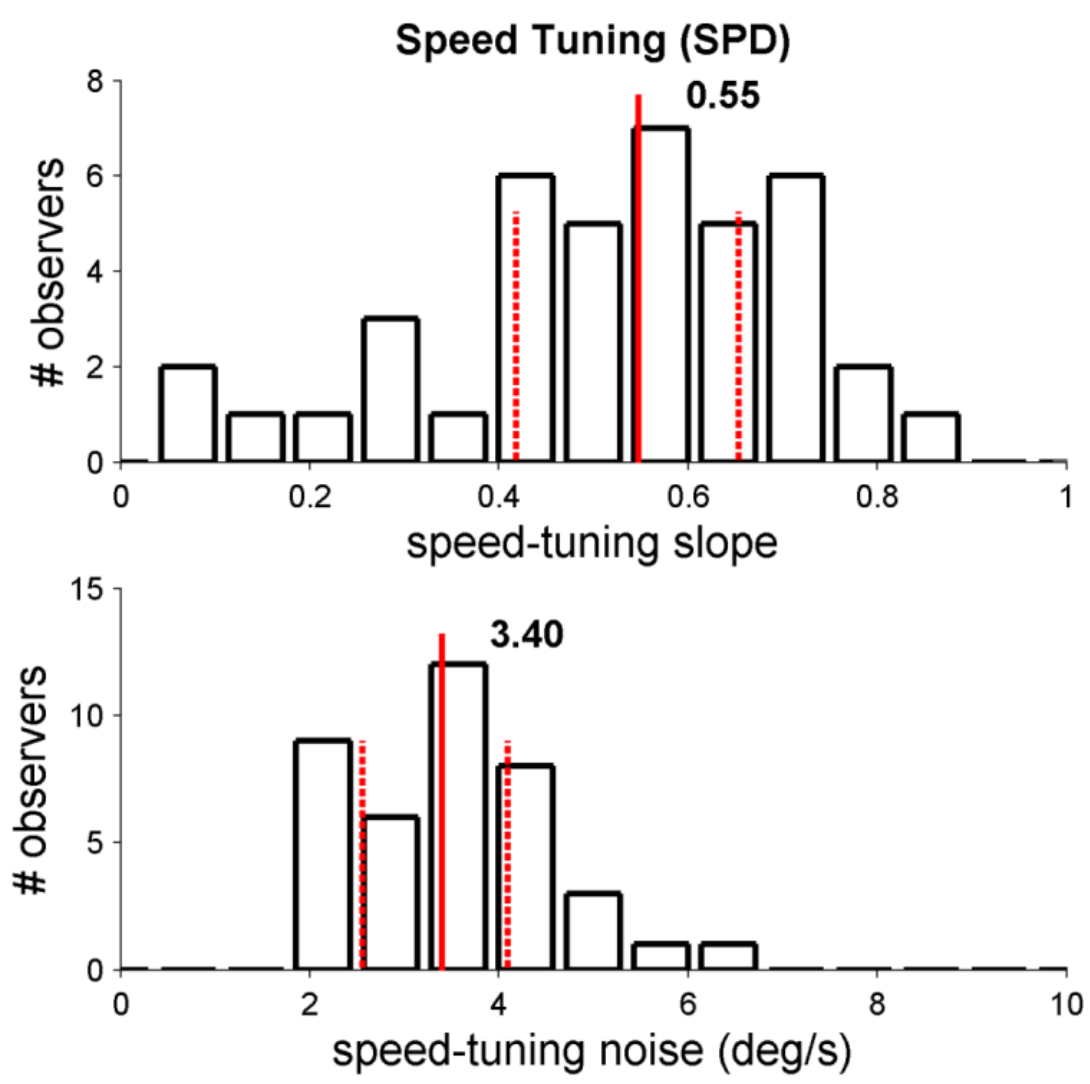

\subsection{Reliability and Across-Time Variability}

The inter-subject variance is a factor of approximately four greater than the test/retest variance, on average, across six candidate metrics (Figure 6). A non-parametric analysis of variance (Kruskal-Wallis test) yielded highly significant effects of subject for all the above metrics at better than the $\mathrm{p}<0.0001$ level, confirming the reliability of these metrics as measures of inter-subject variability and thus their usefulness as performance metrics, even when only a single test session is possible. This is a crucial requirement for any usable clinical test. 

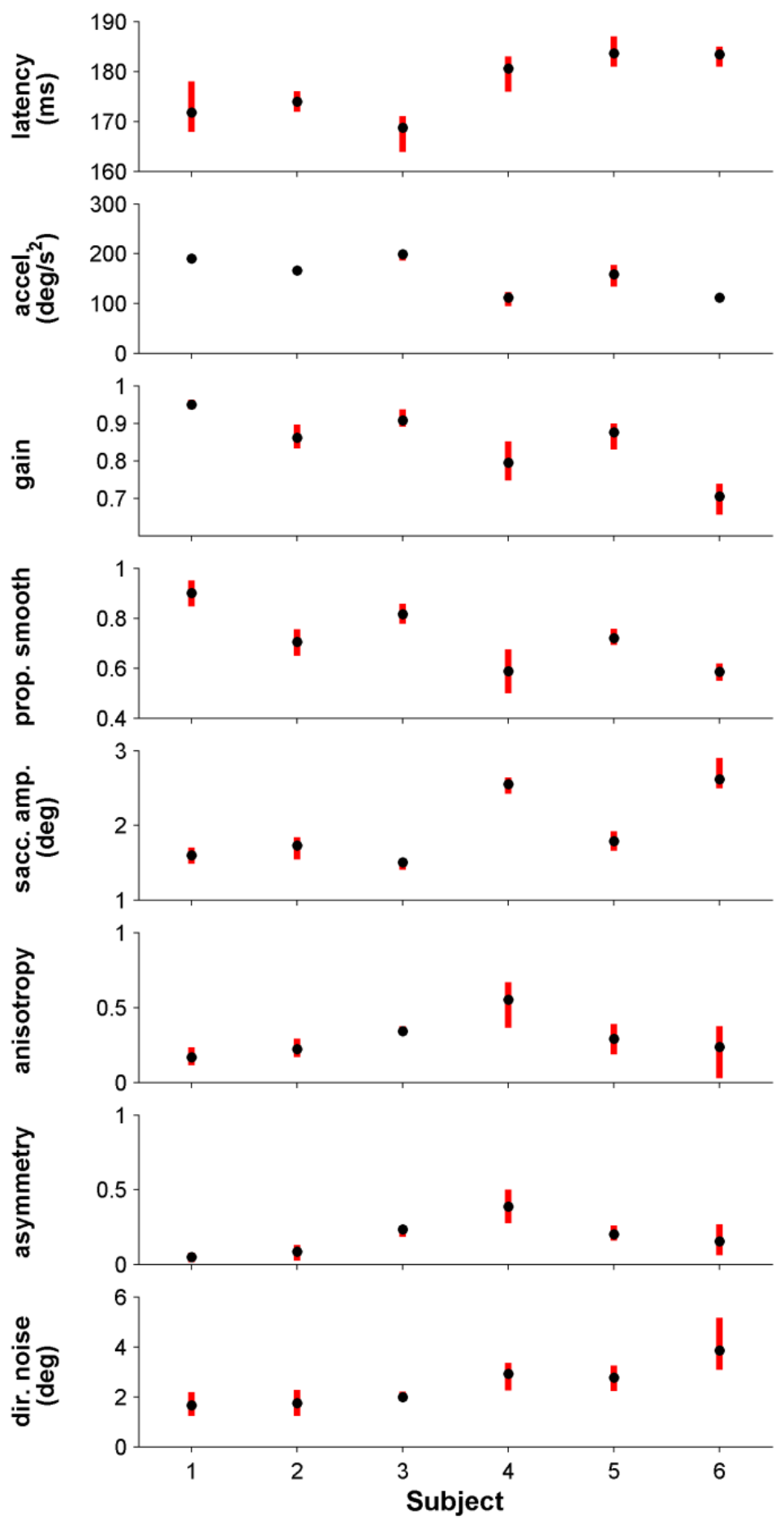


\subsection{Correlation}

This correlation matrix in Figure 7 shows a number of critical findings. First, none of our oculometric measures is correlated with either acuity or age. Thus, they provide novel information about the visual motion processing capabilities of our subjects, rather than visual motion processing impairments due to either acuity or age. Second, the two direction-tuning "nonlinear" metrics (DIRasymmetry, DIRanisotropy) are in a class by themselves. They are not correlated with any of the other measures and, oddly, only weakly correlated with each other. Thus, these two DIR measures provide independent information about the subjects not captured by the other "linear" measures. Third, there is a class of linear metrics that is loosely correlated with each other, although there are some interesting exceptions (e.g., latency is largely uncorrelated with gain). Thus, our 10 oculometric measures provide at least 2 and perhaps as many as 4 largely independent measures of motion processing performance.

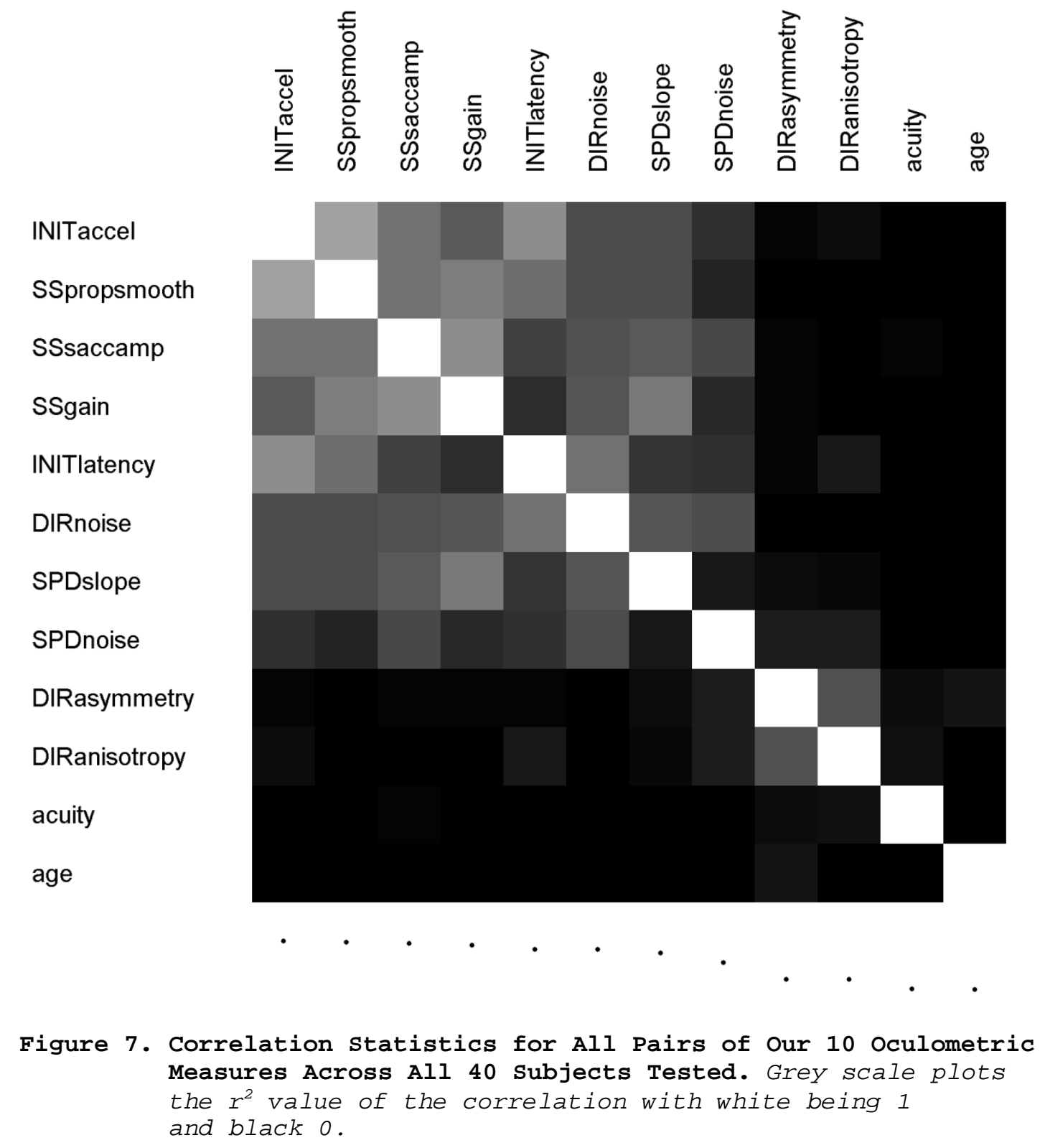




\subsection{CONCLUSIONS}

We have successfully completed all facets of the OMCAT specific aims:

- We have developed, refined, and validated a methodology to collect and compute 10 oculometric measures of human speed and direction perception that could potentially be used as predictors of piloting performance.

- We have demonstrated that reliable measures of this set of metrics can be obtained from a single 15-minute test session using a novel task and efficient data collection.

- We have demonstrated that all 10 of these measures provide information that is uncorrelated with (i.e., independent of) both traditional acuity measures and age.

- We have demonstrated that these measures provide multiple independent dimensions of human motion perception performance (at least two and perhaps as many as four independent classes of oculometrics, each potential predictors of pilot performance).

- We have established a database of the normal distribution of these new performance measures among a healthy group of subjects who would meet existing aircrew vision standards.

\subsection{REFERENCES}

1. Krukowski AE, Stone LS. Expansion of direction space around the cardinal axes revealed by smooth pursuit eye movements. Neuron 2005; 45(2):315-23.

2. Luce RD. Response times: their role in inferring elementary mental organization. New York: Oxford University Press; 1986.

3. Robinson DA, Gordon JL, Gordon SE. A model of the smooth pursuit eye movement system. Biol Cybern 1986; 55(1):43-57.

4. Leigh RJ, Zee DS. The neurology of eye movements. New York: Oxford University Press; 2006.

5. Beutter BR, Stone LS. Human motion perception and smooth eye movements show similar directional biases for elongated apertures. Vision Res 1998; 38(9):1273-86.

6. Liston DB, Krukowski AE, Stone LS. Saccade detection during smooth tracking. Displays 2013; 34(2):171-6.

7. Adler SA, Bala J, Krauzlis RJ. Primacy of spatial information in guiding target selection for pursuit and saccades. J Vis 2002; 2(9):627-44.

8. Carpenter RH. Oculomotor procrastination. In: Fisher DF, Monty RA, Senders JW, eds. Eye movements: cognition and visual perception. Hillsdale, NJ: Lawrence Erlbaum Associates; 1981.

9. Lisberger SG, Westbrook LE. Properties of visual inputs that initiate horizontal smooth pursuit eye movements in monkeys. J Neurosci 1985; 5(6):1662-73. 


\section{LIST OF ABBREVIATIONS AND ACRONYMS}

DIR direct-tuning measures

INIT initiation measures

NASA National Aeronautics and Space Administration

OBVA Operational Based Vision Assessment

OMCAT Oculometric Motion Processing Capability Assessment Tool

SPD speed-tuning measures

SS steady-state measures 